\title{
ADAPTIVE BEAMFORMING AlgORITHM FOR INTERFERENCE SUPPRESSION IN GNSS RECEIVERS
}

\author{
Zhao hongwei $^{1}$, Lian Baowang ${ }^{1}$ and Feng Juan ${ }^{1,2}$ \\ ${ }^{1}$ School of Electronics and Information, Northwestern Polytechnical University, \\ Xian City, P.R.China \\ Hongvi_zhao@hotmail.com \\ ${ }^{2}$ Department of Computer Science, KIT- Karlsruhe Institute of Technology, \\ Karlsruhe, Germany \\ fengeira.uka.de
}

\begin{abstract}
Antenna arrays with space-time adaptive processing (STAP) have been widely used in Global Navigation Satellite System (GNSS) to suppress interference in spatial domain as well as temporal domain, its performance depends on digital beamforming algorithms. In this paper, we propose a joint adaptive beamforming algorithm which produces nullings in the directions of interference while simultaneously maximizing carrier-to-noise ratio $\left(C / N_{0}\right)$ of post-correlation signal. Firstly, the interferences are cancelled by using subspace orthogonal projection. Then the maximum $C / N_{0}$ constraint algorithm is employed to enhance the post-correlation signal. Meanwhile, a simple estimation approach about numbers of interferences is introduced. Finally, simulations demonstrate that using our adaptive algorithm, STAP can effectively suppress the strong interference and improve the capturing capability of GNSS.
\end{abstract}

\section{KEYWORDS}

Adaptive Beamforming, Interference Suppression, Subspace Orthogonal Projection, Max C/No, GNSS Receivers

\section{INTRODUCTION}

Antenna array is a set of antenna elements whose outputs are combined to provide beamforming/nulling steering in specific directions, thus allowing the GNSS receivers to operate in the presence of interference by separate the desired GNSS signals from undesired signals [1]. However, an array of $M$ elements has only $M-1$ degrees of freedom, that is, the number of elements determines the number of interferences can be mitigated effectively. In order to improve the capability of interference suppression, a finite impulse response (FIR) filter is added behind each antenna element, and the resulting set of filters is commonly referred to as a space-time adaptive processing.

The performance of STAP depends on the adaptive beamforming algorithm which controls the adjusting of weight coefficients of filters. Using an adaptive algorithm, the filter weights of the antenna array are obtained according to changes of incident signals. The received signal from each array element is multiplied with the corresponding "weight" coefficient, which varies the amplitude and phase of the signal, and then the weighted signals are combined into one signal so as to achieve better performance over a single antenna element [2].

Many adaptive beamforming algorithms for determine these weights have been proposed over the past decades. The classic adaptive algorithms include least mean square (LMS), recursive

DOI : $10.5121 /$ ijcsit.2011.3502 
least square (RLS), direct matrix inversion (DMI) etc. but for these algorithms require the prior information of incident signal is deterministic, while the prior information is hardly obtained in GNSS applications. Subsequently, some blind adaptive algorithms were proposed such as Power Inversion [3] and subspace orthogonal projection [4], etc., which do not require knowing any prior information about incident signals. Additionally, because adaptive antenna arrays are traditionally used in applications such as radar and wireless communication systems, interference suppression performance is commonly measured by the signal-to-interference-plusnoise ratio (SINR) at the output of the antenna array, and adaptive algorithms tend to be optimized in this regard [7].

However, in the GNSS applications, as the desired GNSS signals are constantly variable and the undesired interferences have random incident direction and magnitude, adaptive array processing is difficult to perform well in both interferences suppression and desired signals enhancement. Previous works focused on the elimination of interferences which resulting in the distortion of desired signals, or focused on the enhancement of desired signals which endure the effect of interferences. To address this problem, we propose a joint adaptive beamforming algorithm for interference suppression in GNSS receivers. The proposed algorithm is a two-step approach: first, subspace orthogonal projection is utilized to suppress strong interferences by producing nulling in array pattern. Then, the maximum post-correlated $\mathrm{C} / \mathrm{N}_{0}\left(\mathrm{MaxC} / \mathrm{N}_{0}\right)$ constraint is used to process the interference-free signal to further enhance the signal quality.

The remainder of this paper is organized as follows. Section 2 introduces some related works about the interference suppression for adaptive antenna array in GNSS applications. Section 3 sets the signal model, which analyzes the STAP model and proposes a design of GNSS anti-jam software receivers. In section 4, we propose the joint adaptive beamforming algorithm. Meanwhile, a simple estimation method of numbers of interferences is addressed. Section 5 conducts the experiments to demonstrate that the performance of interference rejection and enhancing $\mathrm{C} / \mathrm{N}_{0}$ is significantly improved. The conclusion is given in Section 6.

\section{RELATED WORK}

There exist many adaptive algorithms for antenna arrays with different applications. Traditionally, because adaptive antenna arrays are used in applications such as radar and wireless communication systems, interference suppression performance is commonly measured by the signal-to-interference-plus-noise ratio (SINR) at the output of the antenna array, and adaptive algorithms tend to be optimized in this regard [7]. Additionally, some algorithms based on other criterion, such as minimum mean square error (MMSE) [12] and linear constrained minimum variance (LCMV), have also been proposed. However, in the GNSS case, the power of desired signals is well below the noise and interferences, these algorithms are not suitable to apply in GNSS receivers.

The authors in [4] proposed a subspace orthogonal projection algorithm to suppress interference signals by projecting the received signal on the interference-free subspace, where it was shown that the interference mitigation problem can be solved effectively. Nevertheless, after projection, the desired GNSS signals with very low signal-to-noise ratio (SNR) are still merged into noise, which degrade the synchronization performance of GNSS receivers. And, the performance for interference suppression depends on the accuracy of the estimated number of interferences.

In $[5,13]$, the authors addressed an interference cancellation approach based subspace tracking and maximum SNR of the output of antenna array. However, in the GNSS case, the receiver performance is determined by the carrier to noise ratio $\left(\mathrm{C} / \mathrm{N}_{0}\right)$ of post-correlation signal. The authors in [6] show that better signal quality can be obtained using optimized post-correlated $\mathrm{C} / \mathrm{N}_{0}$ compared with optimized SNR. In [7], a comparison of maximum output SNR and 
receiver $\mathrm{C} / \mathrm{N}_{0}$ for adaptive antennas is performed, which demonstrated that output $\mathrm{SNR}$ is an inadequate indication of GNSS receiver $\mathrm{C} / \mathrm{N}_{0}$ performance.

In the paper, we propose a joint adaptive algorithm based on subspace orthogonal projection and maximum post-correlated $\mathrm{C} / \mathrm{N}_{0}$ beamforming. It can mitigate interferences effectively by separate interferences from received signal and enhance GNSS signals significantly by maximum post-correlated signal $\mathrm{C} / \mathrm{N}_{0}$. The details of the proposed adaptive algorithm will be presented in the next sections.

\section{SignAL MODEL}

\subsection{STAP System Model}

Space-time adaptive processing for GNSS receiver is a multi-element antenna array with an adaptive reception pattern, each of which is followed by a proper chain of radio frequency frontend and adaptive FIR filter. Using a special adaptive algorithm, the filter weights of the array are chosen to preserve the desired GNSS signals while suppressing the incident interferences.

Consider a linear antenna array with antennas equidistant along the y-axis as shows in fig.1. The spacing $\mathrm{d}$ between each antenna element is half of the wavelength $\lambda\left(\lambda=c / f_{c}, c\right.$ denotes the speed of light, $f_{c}$ is the carrier frequency). $M$ is the number of antenna elements.

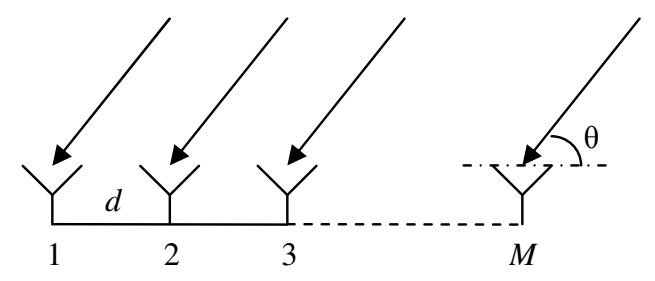

Fig.1. Linear antenna Array with Equi-space

Without loss of generality, assuming the azimuth of all incident signals on antenna array are $\pi / 2$, that is, all signals are from the same plane, the elevation angle of $i^{\text {th }}$ incident signal is $\theta_{i}$. The coordinate vector of the $m^{\text {th }}$ antenna in space is

$$
u_{m}=\left[x_{m}, y_{m}, z_{m}\right]=[0,(m-1) d, 0]
$$

Then, the signal steer vector on array elements can be expressed as

$$
a_{i}=\left[\begin{array}{l}
1 \\
\exp \left\{j \frac{2 \pi}{\lambda}\left(\sin \theta_{i}\right) d\right. \\
\vdots \\
\exp \left\{j \frac{2 \pi}{\lambda}\left(\sin \theta_{i}\right)(M-1) d\right.
\end{array}\right]
$$

The incident signals $\mathbf{x}(n)$ with GNSS signals and interferences, after down-conversion and analog-to-digital (A/D) conversion, can be written as

$$
\mathbf{x}(n)=\sum_{k=1}^{K} s_{k}(n) a_{k}(n)+\sum_{l=1}^{L} j_{l}(n) d_{l}(n)+N(n)
$$

Where, $\mathbf{x}(n)=\left[x^{1}(n), x^{2}(n), \ldots, x^{\mathrm{M}}(n)\right]$, it denote the signal vector received by $M$ elements. $s_{k}(n), a_{k}(n)$ is amplitude and direction vector respectively of the $k^{\text {th }}$ GNSS signal. $j_{l}(n), d_{l}(n)$ is amplitude and direction vector respectively of the $l^{\text {th }}$ interference signal. $K$ and $L$ is the total 
number of received GNSS signal and interference signal separately. $N(n)$ is Gaussian white noise with 0 mean and $\delta^{2} \mathrm{I}_{M}$ variance.

The STAP processor with $M$ elements is modelled as a $P$-tap FIR filter attached to per front-end channel output. The structure can be depicted as fig. 2 [8]. In fig. 2, each complex weight $w_{m p}$ in the STAP filter corresponds to the $p^{\text {th }}$ weight of the $m^{\text {th }}$ filter where $\mathrm{m}$ ranges from 1 to $M$.

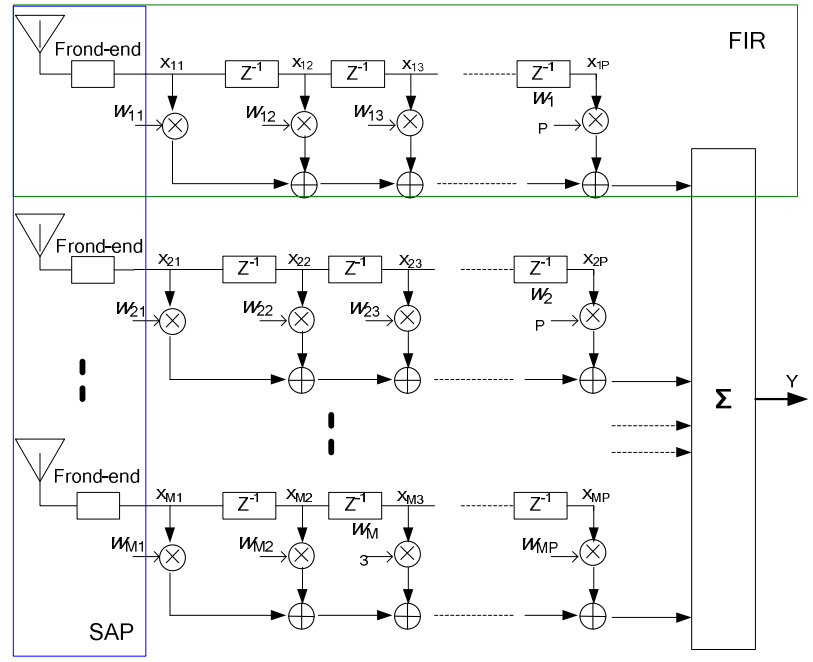

Fig.2. STAP model

The digitized output of the $m^{\text {th }}$ element is denoted by $\boldsymbol{x}^{m}(n)$, and the instantaneous signal snapshot at time $n$ on the taps of the $m^{\text {th }}$ filter can be written as

$$
\boldsymbol{x}^{m}(n)=\left[x^{m}(n), x^{m}(n-1), \ldots, x^{m}(n-P+1)\right]^{T}
$$

Accordingly, the snapshot on each element can be denoted by an $M P x 1$ signal vector

$$
\boldsymbol{X}(n)=\left[\begin{array}{l}
x^{1}(n) \\
x^{1}(n-1) \\
\vdots \\
x^{1}(n-P+1) \\
x^{2}(n) \\
\vdots \\
x^{M}(n-P+1)
\end{array}\right]
$$

Similarly, the weights can be combined into a signal $M P x 1$ weight vector

$$
W=\left[w_{11}, w_{12}, \cdots w_{1 P}, \cdots w_{M 1}, \cdots w_{M P}\right]
$$

Consequently, the output of STAP can be expressed as

$$
\boldsymbol{Y}=W^{H} \boldsymbol{X}
$$

\subsection{GNSS Software Receiver}

The output of STAP is transfer to baseband module in receivers for synchronization processing. Because of the limitation of the sampling rate and processing speed, the adaptive algorithm for STAP is executed at intermediate frequency rate of baseband. In this study, we firstly present a 
design of GNSS anti-jam receiver based on STAP, called STAP/GNSS receiver. The block diagram of software receiver is depicted as fig.3. Where, $M$ is the numbers of frond-ends corresponding to $M$ elements and $N$ is the numbers of channels.

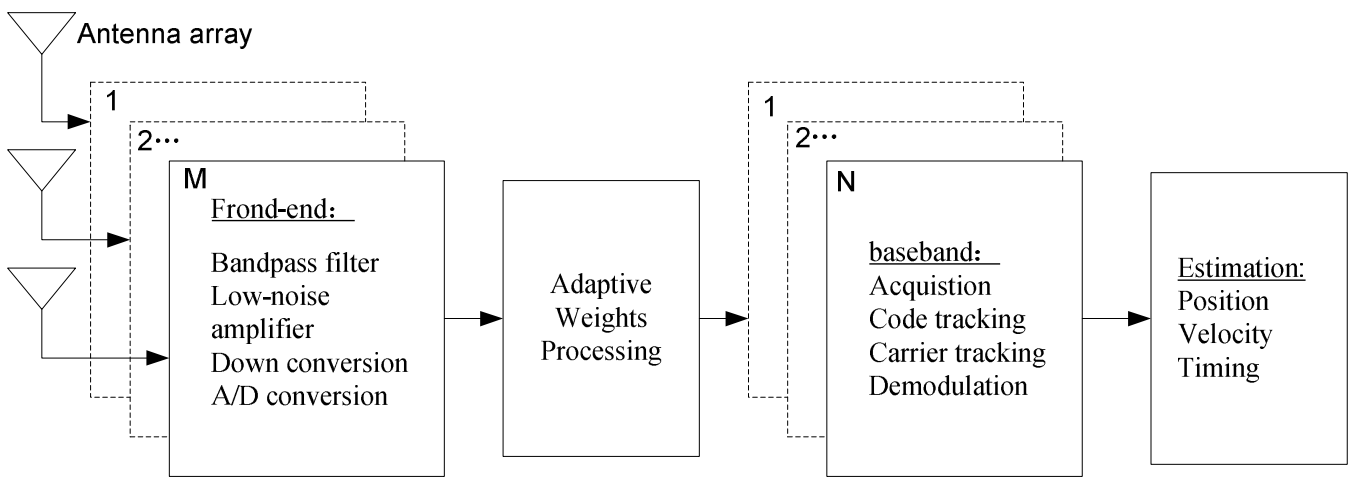

Fig.3. STAP-based GNSS Receiver block diagram

Typically, the adaptive weights processing and baseband processing module are processed in parallel, where the output of front-end be used together.

The received signal multiplied with weights in the stage of adaptive processing, and then enters baseband module for further processing. The traditional block diagram of GNSS baseband processing is showed as fig. 4 .

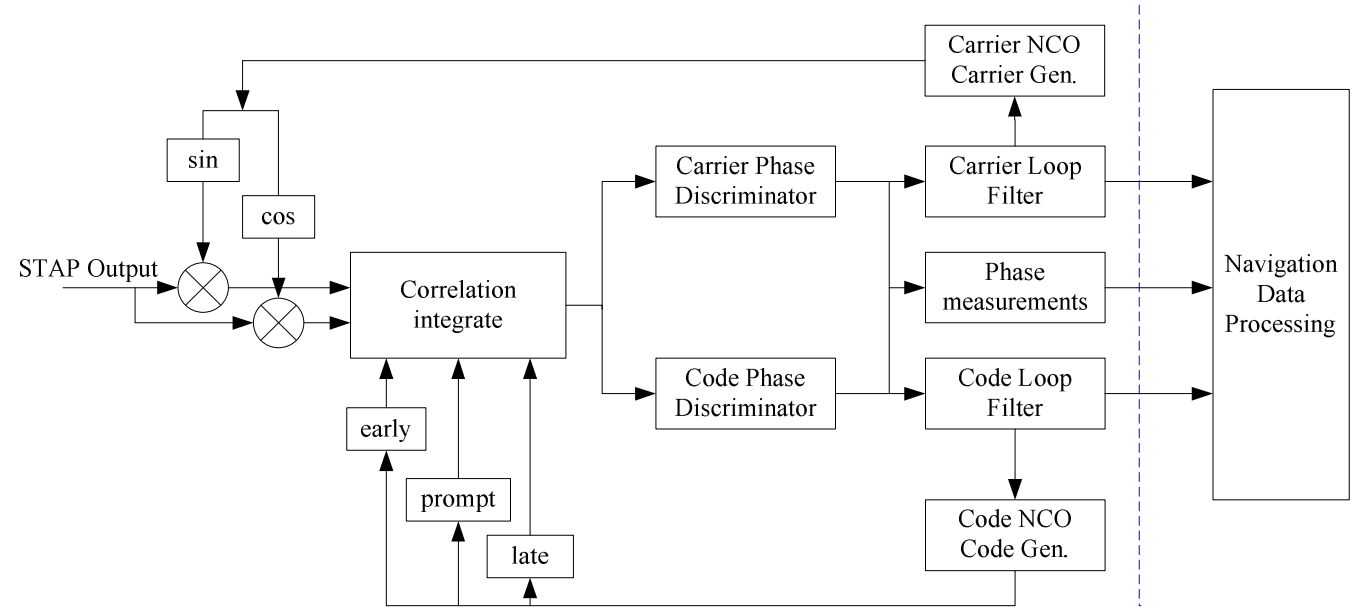

Fig.4 the simplified GNSS baseband processing block diagram

In the baseband module, receiver implements the acquisition and tracking of GNSS signal. The acquisition approach in this design is sliding correlation and the tracking loops adopt the traditional phase lock loop (PLL) and delay-lock loop (DLL).

\section{Proposed Adaptive Beamforming Algorithm}

\subsection{Subspace orthogonal Projection}

Assuming the desired signal is entirely uncorrelated with interferences and background noise. The received signal can be decomposed into two orthogonal subspaces called as signal subspace and interference subspace, respectively. The strong interferences will be cancelled by projecting the received signal onto the signal subspace. 
Assuming the received signal is express as (3), the cross-covariance matrix $R$ of received signal can be denoted by

$$
R_{x x}=E\left\{X(n) X^{H}(n)\right\}=R_{S}+R_{J}+R_{N}
$$

Where $E\{\bullet\}$ denotes the expectation, $(\bullet)^{H}$ denotes the Hermitian transpose, $R s, R_{J}$ and $R_{N}$ are the contributions of GNSS signals, interferences and noise in the overall cross-covariance respectively, and the definitions are as follows:

$$
\begin{aligned}
& R_{s}=E\left\{s(n) s^{H}(n)\right\} \\
& R_{J}=E\left\{j(n) j^{H}(n)\right\} \\
& R_{N}=E\left\{N(n) N^{H}(n)\right\}=\sigma^{2} I
\end{aligned}
$$

The cross-covariance $R_{x x}$ is a positive-definite matrix, it has an eigen decomposition given as

$$
R_{x x}=\sum_{i=1}^{M} \lambda_{i} e_{i} e_{i}^{H}
$$

Here, $\lambda_{i}$ is eigenvalue and arrange them in descending, that is, $\lambda_{1} \geq \lambda_{2} \geq \ldots \lambda_{K}>0 . e_{i}$ is the eigenvector corresponding to eigenvalue $\lambda_{i}$. Because the GNSS signal power is well below background noise and the interferences power is much stronger than that of background noise, that is, interferences are dominating in the overall cross-covariance matrix $R_{x x}$. Rewrite (12) we have

$$
R_{x x}=\sum_{i=1}^{L} \lambda_{i} e_{i} e_{i}^{H}+\delta^{2} \sum_{i=L+1}^{M} e_{i} e_{i}^{H}
$$

Where, the first addend with $L$ bigger eigenvalues corresponds to $L$ strong interferences, and smaller eigenvalues correspond to background noise and desired GNSS signals.

The eigenvectors are orthogonal to one another, and any subset of eigenvectors spans a subspace which is orthogonal to the subspace spanned by the remaining eigenvectors. So, the eigenvectors $e_{1}, e_{2}, \ldots e_{L}$ can be spanned to compose $M$ by $L$ interference subspace, and $e_{L+1} \ldots$, $e_{M}$ form $\mathrm{M}$ by $(M-L)$ signal subspace.

Denoting the projection matrix of signal subspace by $P_{d}$, then

$$
P_{d}=\sum_{L+1}^{M} e_{l} e_{l}^{H}
$$

The interference-free output is

$$
y_{\text {sub }}=X(n)^{H} P_{d}
$$

Inserting (3) into (15), then

$$
y_{\text {sub }}=\left[\sum_{k=1}^{K} s_{k}(n) a_{k}(n)+\sum_{l=1}^{L} J_{l}(n) d_{l}(n)+N(n)\right]^{H} P_{d}
$$

Because of the orthogonality, the strong interference will be effectively suppressed by projecting the received signal into the signal subspace. Consequently, (16) becames

$$
y_{\text {sub }}=\left[\sum_{k=1}^{K} s_{k}(n) a_{k}(n)+N(n)\right]^{H} P_{d}
$$


Let $S_{d}=\sum_{k=1}^{K} s_{k}(n) a_{k}(n)$ denote the desired signals vector, then (17) can be written as

$$
y_{s u b}=\left[S_{d}+N(n)\right]^{H} P_{d}
$$

Note that in the above discussion, there is a prerequisite that the number of interferences must be known before eigen decomposition of cross-covariance matrix $R_{x x}$. That is, it is essential to estimate the number of interferences present in the received signal. Some estimation algorithms have been applied effectively such as Akaike Information Criterion (AIC) and Minimum Description Length (MDL) [9]. Though the two criteria have shown effectiveness in some extent, both tend to overestimate the number of interferences [10].

Because the desired GNSS signals are much weaker than interferences and background noise and $\delta^{2}$ in equation (13) corresponds to the noise power, it is reasonable that the number of those eigenvaluses in equation (13) with significantly greater than $\delta^{2}$ are regarded as the number of interferences. Consequently, in this study, we set a threshold with $5 \mathrm{~dB}$ above $\delta^{2}$ and then count the number of eigenvalues greater than the threshold which is assumed equal to the number of interferences. The simulations have shown the approach is simple and effective.

\subsection{Joint adaptive beamforming algorithm}

After orthogonal projection, the interference-free signal $y_{\text {sub }}$ is still merged into background noise. In order to enhance the interference-free signal, maximum $\mathrm{C} / \mathrm{N}_{0}$ beamforming algorithm is applied to obtain the weights of STAP filter. Combined (7) with (18), the output of STAP becomes

$$
Y=w^{H} y_{\text {sub }}=w^{H}\left[S_{d}+N(n)\right]^{H} P_{d}
$$

Receivers signal acquisition is the process that received signal correlated with local reference code. Take GPS as an example, the sampling interval is $T_{0}$ and integral period is denoted by $T_{0} N_{r}$. The cross-correlation function can be expressed as

$$
R_{y d}(\tau)=\frac{1}{N_{r}} \sum_{n=1}^{N_{r}} Y[n] d\left(n T_{0}+\tau\right)
$$

Where $d(t)$ is the locally generated reference C/A code, $\tau$ is the code phase delay. Inserting (19) into (20) yields

$$
R_{y d}(\tau)=w^{H}\left[\frac{1}{N_{r}} \sum_{n=1}^{N_{r}}\left(S_{d}+N(n)\right) d\left(n T_{0}+\tau\right)\right] P_{d}
$$

Let

$$
\begin{aligned}
& s_{d}(\tau)=\frac{1}{N_{r}} \sum_{n=1}^{N_{r}} S_{d} d\left(n T_{0}+\tau\right) \\
& s_{N}(\tau)=\frac{1}{N_{r}} \sum_{n=1}^{N_{r}} N(n) d\left(n T_{0}+\tau\right)
\end{aligned}
$$

Equation (21) can be written as

$$
R_{y d}(\tau)=w^{H} s_{d}^{T}(\tau) P_{d}+w^{H} s_{N}(\tau) P_{d}
$$

In order to derive the weights of STAP filter that maximizes $\mathrm{C} / \mathrm{N}_{0}$, we use an equivalent equation defined in [11]. 
International Journal of Computer Science \& Information Technology (IJCSIT) Vol 3, No 5, Oct 2011

$$
\mathrm{C} / \mathrm{N}_{0}=\frac{1}{T} \frac{\left|E\left\{R_{y d}(\tau)\right\}\right|^{2}}{\operatorname{var}\left\{R_{y d}(\tau)\right\}}
$$

In (21), the reference code $d(t)$ is a pseudo random noise which has zero mean, and $N(n)$ is white Gaussian noise with 0 mean and $\sigma^{2}$ variance. Therefore, the mean of $R_{y d}(\tau)$ is

$$
E\left\{R_{y d}(\tau)\right\}=E\left\{w^{H} s_{d}^{T}(\tau) P_{d}\right\}
$$

The variance of $R_{y d}(\tau)$ is the noise power at a particular $\tau$. Consequently, equation (26) can be expressed by

$$
\begin{aligned}
\mathrm{C} / \mathrm{N}_{0} & =\frac{1}{T} \frac{\left|E\left\{w^{H} s_{d}^{T}(\tau) P_{d}\right\}\right|^{2}}{\operatorname{var}\left\{R_{y d}(\tau)\right\}} \\
& =\frac{1}{T} \frac{\left|w^{H} s_{d}^{T}(\tau) P_{d}\right|^{2}}{E\left\{\left|w^{H} s_{N}(\tau) P_{d}\right|^{2}\right\}} \\
& =\frac{1}{T} \frac{w^{H} P_{d} s_{d}(\tau) s_{d}^{T}(\tau) P_{d}^{H} w}{\sigma^{2} w^{H} P_{d} P_{d}^{H} w}
\end{aligned}
$$

The correlation matrix of desired signal component is defined as

$$
R_{d}(\tau)=s_{d}(\tau) s_{d}^{T}(\tau)
$$

Therefore, the define of $\mathrm{C} / \mathrm{N}_{0}$ can be rewritten as

$$
\mathrm{C} / \mathrm{N}_{0}=\frac{1}{T} \frac{w^{H} P_{d} R_{d} P_{d}^{H} w}{\sigma^{2} w^{H} P_{d} P_{d}^{H} w}
$$

The optimum weight vector can be found to solve the general eigenvalue problem, it satisfy the eigenvalue equation

$$
\left(P_{d} P_{d}^{H}\right)^{-1} P_{d} R_{d} P_{d}^{H} w_{0}=\lambda w_{0}
$$

Where $w_{0}$ is the eigenvector of $\left(P_{d} P_{d}^{H}\right)^{-1} P_{d} R_{d} P_{d}^{H}$ corresponding to the maximum eigenvalue.

Note that the algorithm is based on subspace orthogonal projection and maximum $\mathrm{C} / \mathrm{N}_{0}$, and the weights are in steady-state during the receiver correlation. The algorithm implementation diagram can be depicted as fig. 5

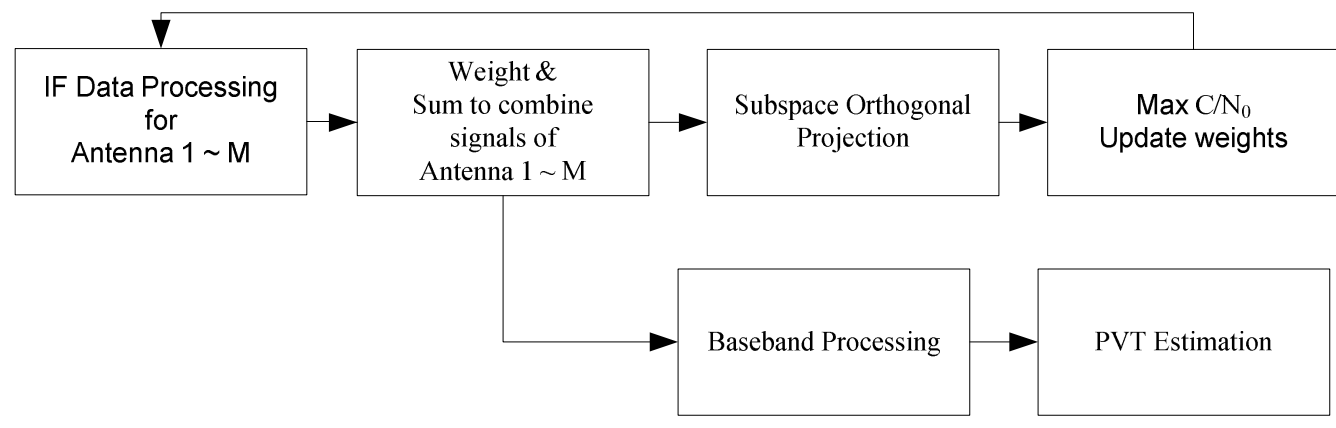

Fig.5 Adaptive beamforming Algorithm flow 


\section{Simulation}

This section demonstrates the advantages of the proposed algorithm. Firstly we build a signal simulation environment that allows generation of GNSS signals on an adaptive antenna array, as well as injection of different types of interference. Then, we examine the performance of interference suppression based on proposed algorithm; meanwhile, the pattern of antenna array is evaluated by utilizing the antenna array shown in Fig.1. Finally, we demonstrate the improved acquisition capability based on a GNSS software receiver.

Consider the simulation scenario as follows. A mixed signal that consists of one GNSS signal with background noise and two interferences reached on antenna array. The elevation angle interference signals are $60^{\circ}$ and $-50^{\circ}$ respectively. Without loss of generality, let the azimuth angle of all incident signals are $\pi / 2$, that is, all signals are from the same plane. The SNR of GNSS signal is $-20 \mathrm{~dB}$ and the interference-to-signal ratio (ISR) is $40 \mathrm{~dB}$. The spectrum of mixed signal is shown as figure 6 .

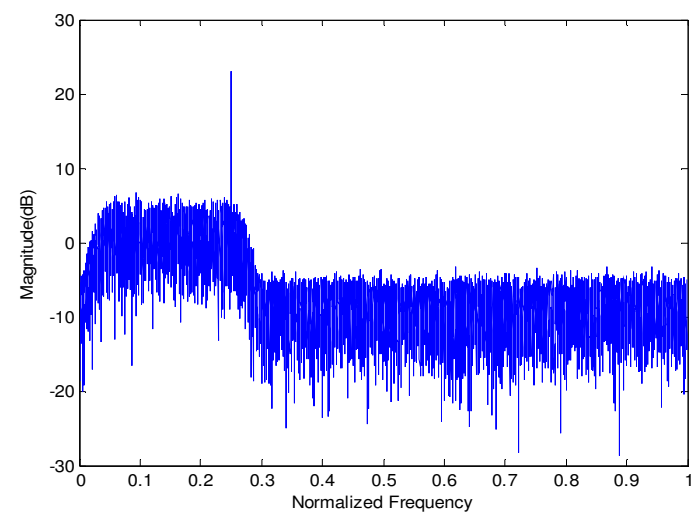

Fig.6 spectrum of mixed signal

In this study, a uniform linear array with 8-element is utilized, the time-tap of FIR for each channel is set to 7. Assuming the carrier of received signal has been stripped, and the pseudorandom noise $(\mathrm{PN})$ code chip rate is $1.023 \mathrm{MHz}$. The sampling frequency and sampling time are set to $4.092 \mathrm{MHz}$ and $1 \mathrm{~ms}$ respectively.

In the first experiment, we investigate the interference suppression performance based on subspace orthogonal projection. Fig.7 shows that the array can adaptively generate nullings at interference direction. Accordingly, the interference signal is mitigated effectively.

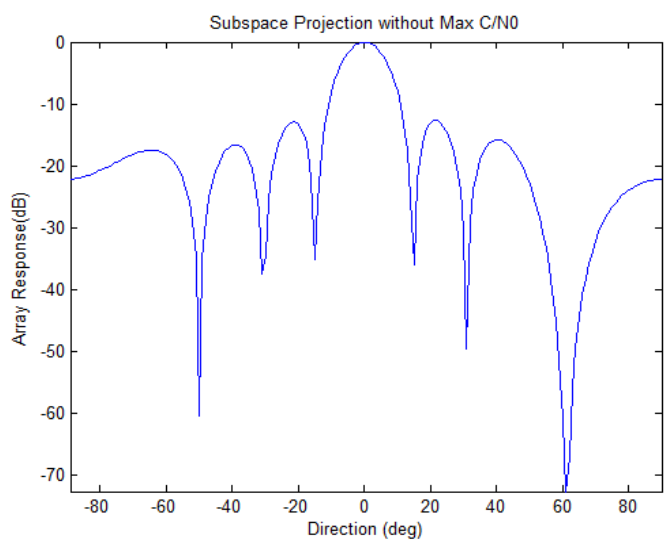

Fig.7 beamforming pattern using subspace orthogonal projection 
In the second experiment, we demonstrate the interference suppression and GNSS signal synchronization capability can be significantly improved by using proposed algorithm. The synchronization process of GNSS signal is accomplished though the acquisition of the Doppler frequency shift and PN code phase delay. In this simulation, the normalized Doppler frequency shift is assumed to be 0.01 and the PN code phase delay is 2000 sample points.

Fig. 8 shows the array pattern based on the proposed algorithm. From Fig. 8 we can see that the deeper nullings are generated at interference directions compare with Fig.7 so as to mitigate interferences more effectively. Moreover, the pattern gain at other directions is balanced which has a minimal effect on desired signals.

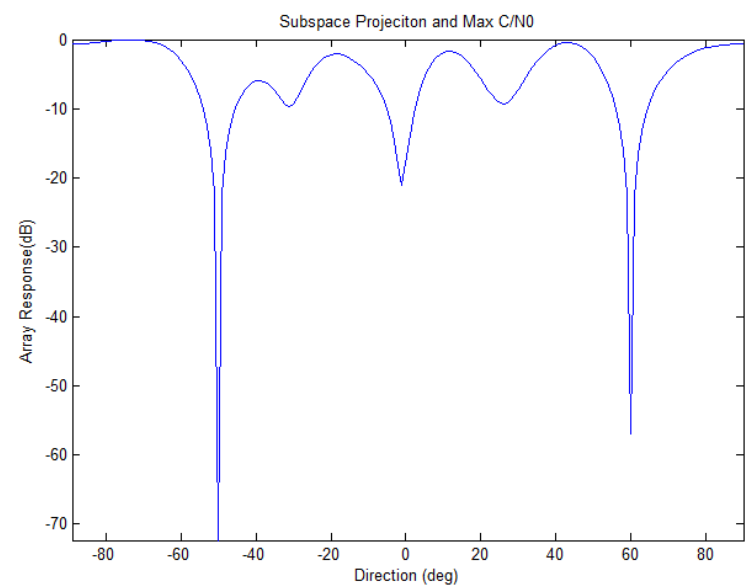

Fig.8 beamforming pattern using proposed algorithm

Fig.9 show the cross-correlation value between local PN code and the output of STAP which is only processed by subspace orthogonal projection. We can see from fig.9 that the peak of correlation value is well-marked, but the influence of noise is still great which degrade the acquisition and tracking capability of GNSS receivers.

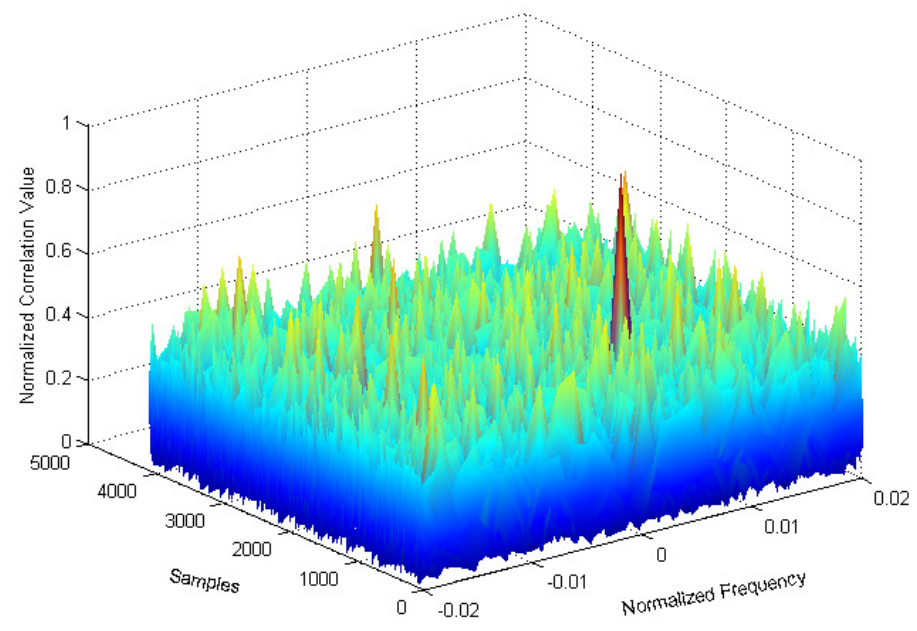

Fig.9 Cross-correlation with subspace orthogonal projection 
When subspace orthogonal projection and Max $C / \mathrm{N}_{0}$ are used together, a significant peak in cross-correlation value as shown in Fig. 10 is present, and the peak occurs at the correct location. Compare Fig. 10 with Fig.9, it can be conducted that the proposed algorithm enhances desired signal quality significantly while suppress interferences effectively.

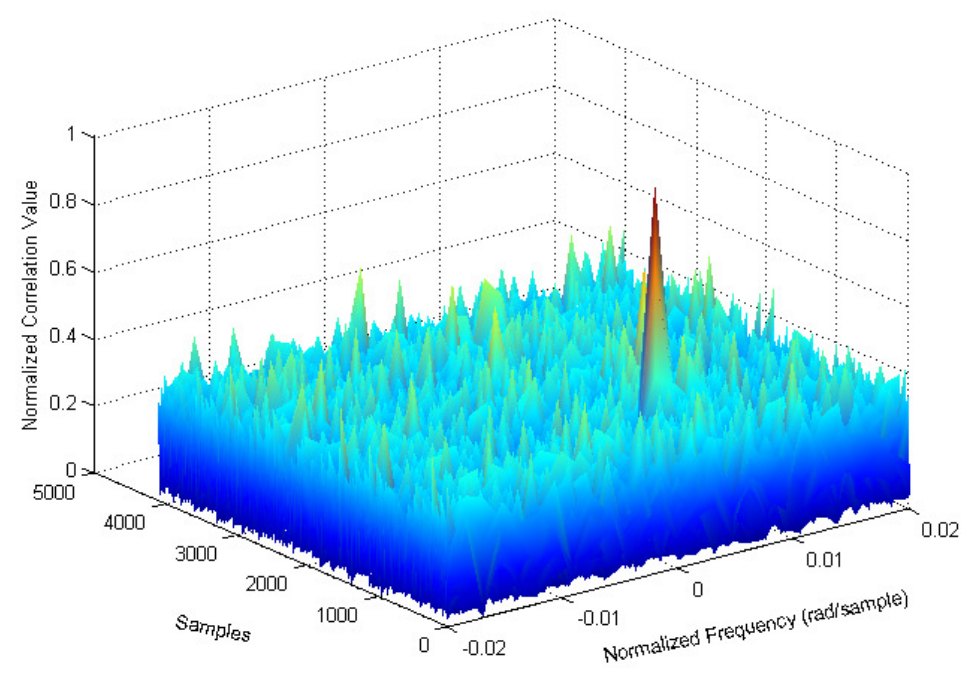

Fig.10 Cross-correlation with subspace orthogonal and $\mathrm{Max} C / \mathrm{N}_{0}$

\section{Conclusions}

In this paper, we proposed a joint adaptive beamforming algorithm to suppress interference and to enhance $\mathrm{C} / \mathrm{N}_{0}$ of post-correlation signal. The strong interference can be effectively cancelled by using subspace orthogonal projection, and then, a maximum $\mathrm{C} / \mathrm{N}_{0}$ beamforming was used to enhance the desired signal. In order to validate the algorithm, continued wave interference and wideband interference are introduced in simulation scenario. The experimental results show that the significant performance of proposed approach and, compare to simplex subspace orthogonal projection, the proposed approach improves the signal acquisition and tracking capability of GNSS receiver.

\section{ACKNOWLEDGEMENTS}

The work was supported in part by the National Defence key Laboratory of Integrated Avionics System of China and by a grant from the Aviation Science Foundation under program No. 20085553016.

\section{REFERENCES}

[1] R. Fante \& J. Vaccaro, (2000) "Wideband cancellation of interference in a GPS receive array", IEEE Transactions on Aerospace and Electronic Systems, Vol. 36, No. 4, pp549-564.

[2] I. J. Gupta \& T. D. Mppre, (2004) "Space-frequency adaptive processing for radio frequency interference mitigation in spread-spectrum receivers", IEEE Transactions on Antennas and Propagation, Vol.52, No.6, pp1611-1616.

[3] SEUNG-JUN KIM \& RONALD A.ILTIS, (2004) "STAP for GPS Receiver Synchronization", IEEE Transactions on Aerospace and Electronic System, vol. 40, No.1, Jan. 2004.

[4] W.Utschick, (2002) "Tracking of signal subspace projectors", IEEE Transactions on Signal Processing, Vol.50, No.4, pp.769-778. 
International Journal of Computer Science \& Information Technology (IJCSIT) Vol 3, No 5, Oct 2011

[5] R. Wang, M. Yao, Z. Cheng \& H. Zou, (2011) "Interference cancellation in GPS receiver using noise subspace tracking algorithm”, Signal Processing, Vol.91, N0.2, pp338-343.

[6] John W. Betz, (2001) "Effect of partial-band interference on receiver estimation of $\mathrm{C} / \mathrm{N}_{0}$ : Theory", Proceedings of ION 2001 National Technical Meeting..

[7] Andrew J. O'Brien, Inder J. Gupta, (2009) “Comparison of Output SINR and Receiver C/N $\mathrm{N}_{0}$ for GNSS adaptive Antennas", IEEE Transactions on Aerospace and Electronic Systems, Vol.45, No.4.

[8] Zhao Hongwei, Lian Baowang \& Feng Juan, (2011) "Interference suppression in GNSS receiver using space-time adaptive processing”, $3^{\text {rd }}$ International Conference on Communication Software and Networks.

[9] Eran Fishler, Michael Grosmann \& Hagit Messer, (2002) "Detection of signals by information theoretic criteria: General Asymptotic Performance Analysis", IEEE Transactions on Signal Processing, Vol. 50, No. 5.

[10] Y.Zheng, (2008) "Adaptive antenna array processing for GPS receivers" [D], the University of Adelaide.

[11] J. W. Betz, (2000) "Effect of narrowband interference on GPS code tracking accuracy", In proceedings of ION 2000 National Technical Meeting.

[12] D. Guo, S. Shamai, and S. Verdu, (2011) "Estimation in Gaussian Noise: Properties of the Minimum Mean-Square Error”, IEEE Transactions on Information Theory, Vol.57, No.4.

[13] Y. Hou, W. Guo, X. Jin, (2010) "Design of an anti-jamming GPS receiver based on orthogonal projection method”, Journal of Systems Engineering and Electronics, Vol.21, No.1, pp.16-19.

\section{Authors}

Zhao Hongwei, Ph.D student

Research field, satellite communication, navigation.
Lian Baowang, Professor

Research field, Satellite navigation, wireless communication and embedded system 